\title{
COHOMOLOGY, SYMMETRY, AND PERFECTION
}

\author{
EMIL: BifEr*
}

\begin{abstract}
We explain the philosophy behind the computations in [BDP] and place them in a wider conceptual setting. We also outline, for toric varieties, the resulting equivariant approach to some key results in that theory.
\end{abstract}

\section{Symmetry}

In many situations that arise in Algebraic Geometry one is interested in computing the multiplicative structure of the cohomology ring $H^{*}(X)$, with rational coefficients say, of some algebraic variety $X$. Examples of such situations include toric varieties, complete quadrics, complete symmetric varieties [DP1-2], ... Sometimes, as in the examples just mentioned, the variety $X$ is endowed with symmetries that reflect the action of some algebraic group $G$ on it. In these cases there is a recipe; inspired by the work of M. F. Atiyah and R. Bott [ABI], that often works:

1. Find a strongly $G$-perfect decomposition of $X$ (cf. Section 3 below for a precise statement.) In the examples above, this is simply the decomposition into orbits. In general there is a natural candidate: the Kempf-Hesselink stratification of $X[\mathbf{H}],[\mathrm{K}],[\mathrm{N}]$, a natural outgrowth of D. Mumford's Geometric Invariant Theory.

2. With the help of this decomposition compute the equivariant cohomology ring $H_{G}^{*}(X)$. (At this point it may also be natural to apply the machinery of the localization theorem [AB2], [Hs]; in so doing one usually obtains other interesting descriptions of $H_{G}^{*}(X)$.)

3. Recover $H^{*}(X)$ from $H_{G}^{*}(X)$.

\footnotetext{
*This paper is dedicated to the memory of my friend Pere Menal. I chose the present topic for this occasion because it was the subject of our last conversation. I miss him very much.
} 
This recipe is just one more instance of the old philosophy of using any symmetries that may be present in the problem in order to simplify it.

We shall work, for simplicity, with equivariant cohomology defined in terms of homotopy quotients i.e. the Borel construction (sce Section 2 below.) But the knowledgeable reader could substitute throughout $H_{G}^{*}(X)$ by the smooth $\ell$-adic cohomology of the algebraic stack determined by the $G$-varicty $X$. He would thus gain the advantage of having a completely algebraic theory with, as a bonus, an interesting arithmetic twist [B2].

In the examples mentioned earlier, the recipe works and gives very explicit results. We shall consider below in some detail the case of toric varieties, but the reader is advised to look at [BDP] for a thorough treatment of these examples from the present point of view. In fact one of the aims of this paper is to better explain the philosophy behind those computations and to place them in a wider conceptual setting.

Another aim of the paper is to outline in the last section an "equivariant" approach to some key results in the theory of toric varieties. This approach clarifies, I believe, the nature of these results.

The text of the first three sections follows closely a talk delivered at the University of Copenhagen in July 1989 on the occasion of the Zeuthen Symposium. I would like to thank S. Kleiman and A. Thorup for organizing that conference and creating a very friendly athmosphere.

\section{Cohomology}

Suppose that a topological group $G$ acts properly on a space $X$. The (equivariant) cohomology of the $G$-space $X$ should be the cohornology of the quotient $X / G$. Unfortunately, unless $G$ is acting freely on $X$, the usual topological quotient does not provide a useful theory. In general, it is nocessary to find the right notion of quotient. The best notion is probably obtained by taking the quotient in the 2-category of toposes [SGA4]. However, for simplicity, we shall work here with a homotopy quotient $X_{G}$. (In a purely algebraic context the role of $X_{G}$ would be played by the algebraic stack determined by the $G$-variety $X$; the equivariant cohomology would just be the smooth $\ell$-adic cohomology of this stack. ) Before we can describe this notion of quotient, however, it is necessary to look closely at the case where $X$ is a point i.e. the theory of characteristic classes.

Recall that a classifying space for principal $G$-bundles is by definition a space $B G$ together with a universal principal $G$-bundle $E G$ over it. By universal we mean that isomorphism classes of principal $G$-fibre bundles 
over a nice space $X$ correspond naturally to homotopy classes of maps from $X$ to $B G$. The correspondence is given by pulling-back the universal bundle $E G$. The cohomology ring $H^{*}(B G)$ is by definition the ring of characteristic closses of $G$.

Example 1. $G=\mathrm{C}^{\times}$. This is simply the theory of line bundles, and the classifying space is the infinite projective space $P \mathcal{C}$. Its cohomology ring $H^{*}(B G)=\mathrm{Z}\left[c_{1}\right]$ is a polynomial ring in one variable of degree two.

Example 2. $G=T=\mathrm{C}^{\times} \times \cdots \times \mathrm{C}^{\times}$(an algebraic torus.) In this case we have:

$$
B T \simeq B C^{\times} \times \cdots \times B C^{\times}
$$

and its cohomology is a polynomial algebra in several variables (as many as factors.) In fact, if $X(T)$ denotes the group of algebraic characters of $T$, then

$$
H^{*}(B T) \simeq \operatorname{Sym}^{*} X(T) .
$$

In particular $H^{2}(B T) \simeq X(T)$.

Example 3. $G=\mathrm{GL}_{n}(\mathrm{C})$. The classifying space is the infinite dimensional Grassmannian and

$$
H^{*}(B G)=\mathrm{Z}\left[c_{1}, \ldots, c_{n}\right]
$$

where the variables $c_{i}, 1 \leq i \leq n$, have degree $2 i$ and correspond to the Chern classes.

In general, if $T$ is a maximal torus in $G$, we have (with rational coefficients )

$$
H^{*}(B G)=H^{*}(B T)^{W}
$$

where $W=N_{G}(T) / T$ is the Weyl group of $(G, T)$ and the right hand side denotes the subring of invariants.

We are now ready to describe the homotopy quotient mentioned earlier. This is given by the Borel construction $X_{G}$ obtained after exchanging the fibre $G$ of the universal bundle $E G$ with $X$ i.e.

$$
X_{G}=E G \times_{G} X=(E G \times X) / G
$$

where $G$ acts by $g \cdot(e, x)=\left(e g^{-1}, g x\right)$.

Definition. The equivariant cohomology $H_{G}^{*}(X)$ is by definition the cohomology of the Borel construction $X_{G}$.

Note that there is a fibration

$$
X \rightarrow X_{G} \rightarrow B G .
$$


The spectral sequence of this fibration is the key to the third step in the recipe. This is based on work of P. Deligne [De], V. A. Ginzburg $[G]$, F. Kirwan $[\mathbf{K}], \ldots$

Here follow some other properties of equivariant cohomology with rational coefficients $[\mathrm{Hs}],[\mathrm{AB} 2]$ :

a) If $G$ acts freely on $X$, then $H_{G}^{*}(X)=H^{*}(X / G)$.

b) If $T^{\prime}$ is a maximal torus in $G$ and $W=N_{G}(T) / T$ is the Weyl group, then

$$
H_{G}(X)=H_{T}^{*}(X)^{W}
$$

where the right hand side is the subring of $W$-invariants.

c) If $X$ has a single orbit, then

$$
H_{G}^{*}(X) \simeq H^{*}(B H)
$$

where $H$ is the stabilizer of any point.

d) If $K$ is a maximal compact subgroup of $G$, then

$$
H_{G}^{*}(X) \simeq H_{K}^{*}(X) .
$$

One of the reasons equivariant cohomology is easier to compute than ordinary cohomology is that it has many more "points". Let me try to explain this. Most succesful calculations of cohomology achieve their objective by expressing the cohomology of the space under consideration (e.g. projective space $\mathrm{P}^{n}$ ) in terms of that of spaces for which it is already known (e.g. cclls.) Ultimately, however, they reduce the computation to that of the cohomology of a point. If one thinks of ordinary cohomology as being the case $G=1$ of the equivariant one, then it is clear that the points coincide with the orbits. Thus in the equivariant theory every orbit gives rise to a "point", and there are as many points as there are conjugacy classes of subgroups in $G$. The equivariant cohomology of such a point $H$ is precisely the ring of $H$-characteristic classes i.c. the cohomology of the classifying space of $H$. It follows that in the equivariant theory there is much more freedom of movement.

Another important feature of equivariant cohomology is that there is a theory of equivariant Chern classes. A $G$-linearization of a vector bundle $F$ over $X$ is an action $u: G \times F \rightarrow F$ which is lincar on the fibres and turns the projection $\pi: F \rightarrow X$ into a $G$-equivariant map i.e. $\pi(g \cdot x)=g \cdot \pi(x)$ for every $g \in G$ and every $x \in F$. Note that the homotopy quotient $F_{G}$ provides us with a vector bundle over $X_{C}$. The equivariant Chern classes of $(F, u)$ are by definition the Chern classes of $F_{G}$. This takes a most simple form for a line bundle over an orbit. In this case the equivariant Chern class $c(L, u)$ is determined by the 
isotropy action (character) of the stabilizer on the fibre of $L$ over the point. Actually these notions find thcir most natural formulation when expressed in terms of algebraic stacks. For example a $G$-linearized $\mathcal{O}_{X^{-}}$ module is simply a module for the structure sheaf of the algebraic stack determined by the $G$-variety $X$.

\section{Perfection}

Let $X$ be a smooth complex algebraic variety, and let the algebraic group $G$ act on $X$. Suppose $S \subset X$ is a closed $G$-invariant smooth subvariety and let $U=X-S$ be the complementary open set. Under these conditions, there is a long exact sequence (the equivariant ThomGysin sequence, see [AB1] for example)

$$
\cdots \rightarrow H_{G}^{i-2 \operatorname{codim} S}(S) \stackrel{\xi_{S}^{i}}{\longrightarrow} H_{G}^{i}(X) \rightarrow H_{G}^{i}(U) \rightarrow \ldots
$$

Moreover the composite of the maps

$$
\begin{gathered}
H_{G}^{2-2 \operatorname{codim} S}(S) \stackrel{\xi_{S}^{i}}{\longrightarrow} H_{G}^{i}(X) \\
\downarrow_{\text {restriction }}^{i} \\
H_{G}^{i}(S)
\end{gathered}
$$

is multiplication by the Euler class $e\left(N_{S / X}\right)$ (= top Chern class in this context) of the normal bundle $N_{S / X}$.

If this long exact sequence splits into short exact sequences

$$
0 \rightarrow H_{G}^{i-2 \operatorname{codim} S}(S) \rightarrow H_{G}^{i}(X) \rightarrow H_{G}^{i}(U) \rightarrow 0
$$

then, for example, we have

$$
b_{G}^{i}(X)=b_{G}^{i}(U)+b_{G}^{i-2 \operatorname{codim} S}(S)
$$

and one can deduce the equivariant Betti numbers of $X$ from those of $S$ and $U$.

In [AB1] Atiyah and Bott made the following fundamental observation:

If $e\left(N_{S / X}\right)$ is not a zero-divisor in the ring $H_{G}^{*}(S)$, then the morphisms $\xi_{S}^{i}$ are injective and the long exact sequences (3.1) split into short exact sequences (3.2).

This motivates: 
Definition. We say that a decomposition

$$
X=S_{1} \cup \ldots \cup S_{N}
$$

is strongly $G$-perfect if:

1) Each $S_{i}$ is both smooth and $G$-invariant.

2) For cach $k$,

$$
X_{k}=S_{1} \cup \cdots \cup S_{k}
$$

is an open subset of $X$.

3) For each pair $\left(S_{k}, X_{k}\right), k>1$, the Euler class $e\left(N_{S_{k}} / X_{k}\right)$ is a non-zero divisor.

In [AB1] a decomposition is defined to be $G$-perfect if the long exact sequence determined by cach pair $\left(S_{k}, X_{k}\right)$ splits into short exact sequences. In this case one has an identity of equivariant Poincaré series

$$
P_{t}^{G}(X)=\sum_{1 \leq i \leq N} t^{2 \cdot \operatorname{codim} S_{i}} \cdot P_{t}^{G}\left(S_{i}\right)
$$

It is clear that strongly $G$-perfect implies $G$-perfect.

An immediate consequence of the definition is

Proposition. If $\left\{S_{2}\right\}_{1 \leq i \leq N}$ is a strongly $G$-perfect decomposition of $X$, then for every portial union $X_{k}$ the morphism induced by the restrictions to the strota

$$
H_{G}^{*}\left(X_{k}\right) \rightarrow \prod_{1 \leq i \leq k} H_{G}^{*}\left(S_{i}\right)
$$

is injective.

Proof: For $k=1$, it is obvious. Suppose it holds for $k-1$; it suffices to show that the morphisms

$$
H_{G}^{*}\left(X_{k}\right) \rightarrow H_{G}^{*}\left(X_{k-1}\right) \times H^{*}\left(S_{k}\right)
$$

are injective. Consider the diagram

$$
H_{G}^{i-2 \operatorname{codim} S_{k}}\left(S_{k}\right) \stackrel{\xi}{\longrightarrow} \underset{\substack{\downarrow \\ H_{G}^{i}\left(S_{k}\right) .}}{H} H_{G}^{i}\left(X_{k-1} \cup S_{k}\right) \stackrel{\eta}{\longrightarrow} H_{G}^{i}\left(X_{k-1}\right)
$$


Now, if $\eta(a)=0$, then there is a $b \in H_{G}^{i-2 \operatorname{codim} S_{k}}\left(S_{k}\right)$ such that $a=\xi(b)$. But, from

$$
0=(a)=(\xi(b))=b \cup e\left(N_{S_{k} / X_{k}}\right)
$$

it follows that $b=0$ and therefore $a=\xi(b)=0$.

Thus, in principle, if one knows the cohomology rings of the strata and one controls the injection above, it is possible to describe the cohomology ring of $X$. This is the reason we singled out this notion for special consideration.

Atiyah and Bott. also give an infinitesimal criterion for $e\left(N_{S / X}\right)$ to be a non-zero divisor (sce [AB1 Proposition 13.4.]) It is proved in $[\mathrm{K}]$ using this criterion that the Kempf-Hesselink stratification $[\mathrm{H}]$ of a $G$-variety is strongly $G$-perfect in the above sense.

Let us enunciate this last criterion in the case of an orbit:

Proposition. Let $\mathcal{O}$ be a $G$-orbit in the smooth algebraic variety $X$. Choose a point $P \in \mathcal{O}$ and identify $\mathcal{O}$ with $G / G_{P}$, where $G_{P}$ is the stabilizer of $P$. If the isotropic action of a maximal torus $T$ in $G_{P}$ on the normal space $N_{\mathcal{O} / X}(P)$ has no non-zero fixed points, then $e\left(N_{\mathcal{O} / X}\right)$ is a non-zero divisor.

Proof: Since

$$
H_{G}^{*}(\mathcal{O})=H_{G_{P}}^{*}(P) \hookrightarrow H_{T}^{*}(P)
$$

is an embedding; it suffices to see that $e=e\left(N_{O / X}\right)$ is non-zero in $H_{T}^{*}(P)$. But, if

$$
N_{O / X}(P)=\bigoplus_{\chi_{i} \in X(T)} C_{\chi_{*}}
$$

is the weight decomposition, then

$$
e=\prod_{i} \chi_{i} \neq 0 .
$$

since all $\chi_{i}$ are non-zero.

The considerations above motivate:

Definition. We say that $X$ is a perfect embedding (regular cmbedding in [BDP], but this was a bad choice to which I plead guilty) provided

a) Each orbit closure $\overline{\mathcal{O}}$ is smooth and it is the transversal intersection of the codimension one orbit closures that contain it.

b) For every $P \in \mathcal{O}$, the stabilizer $G_{P}$ has a dense orbit in the normal space $N_{\mathcal{O} / X}(P)$.

To any perfect embedding $X$ we associate a simplicial complex $\mathcal{C}_{X}=$ $(V, S)$ as follows: 
1. $V=\left\{v \mid \mathcal{O}_{v}\right.$ is an orbit of codimension one $\}$.

2. $\Gamma \subset V$ is a simplex if, and only if,

$$
\bigcap_{v \in \Gamma} \overline{\mathcal{O}_{v}} \neq ?
$$

(Note that? is a simplex.)

It is easy to show that the simplexes are in one-to-one correspondence with the orbits.

It is clear that the decomposition of $X$ into orbits is in this case strongly $G$-perfect. The algebraic varieties mentioned above, namely toric varieties and complete symmetric varieties (in particular complete quadrics), provide examples of perfect embeddings. In [BDP] an explicit description, based on these ideas, is given for the equivariant cohomology ring of any perfect embedding. It should be possible to extend these results to the case of a well behaved $G$-varicty and the Kempf-Hesselink stratification.

\section{An example: toric varieties}

We shall illustrate the generalities of the preceding sections with the concrete case of toric varieties.

Let $T$ be an algebraic torus. A toric variety is a normal algebraic $T$-variety $X$ containing $T$ as a dense orbit (this includes the requirement that the stabilizer at the points of this orbit be trivial.) References $[D],[F],[O]$ provide very good expositions of the basic theory of these varieties.

A simple example is the affine plane with the action of $T=\mathrm{C}^{\times} \times \mathrm{C}^{\times}$ given by

$$
\left(t_{1}, t_{2}\right)\left(x_{1}, x_{2}\right)=\left(t_{1} x_{1}, t_{2} x_{2}\right) .
$$

This action has four orbits, namely the origin, the two puncturcd axes and the torus $T$ itself. This can be generalized to the action

$$
t \cdot\left(x_{1}, x_{2}\right)=\left(t^{\chi_{1}} x_{1}, t^{\chi_{2}} x_{2}\right)
$$

determined by any integral basis $\left\{\chi_{1}, \chi_{2}\right\}$ of the character group $X(T) \simeq$ $\mathrm{Z}^{2}$. This action induces an action on the ring of regular functions on the affine plane, the ring of polynomials in two variables, given by $(t \cdot f)(x)=$ $f\left(t^{-1} x\right)$. The weight functions (i.e. the functions $f$ such that $t \cdot f=t^{x} f$ for some character $\chi$ called the weight of $f$ ) are precisely the monomials $a \cdot X_{1}^{n_{1}} X_{2}^{n_{2}}$ and their weight is $n_{1}\left(-\chi_{1}\right)+n_{2}\left(-\chi_{2}\right)$. These weights span a 
conc $\sigma^{\vee}$ in $X(T) \otimes R$. Conversely we can recover the variety, including the $T$-action, by taking the Spectrum of the monoid algebra $\mathrm{C}\left[\sigma^{\vee} \cap X(T)\right]$ or, what is essentially the same, the algebra homomorphisms from $\mathrm{C}\left[\sigma^{\vee} \cap\right.$ $X(T)]$ to $\mathrm{C}$.

In general, affine toric varieties can be constructed as follows. We denote

$$
Y(T)=\operatorname{Hom}\left(\mathrm{C}^{\times}, T\right)
$$

the group of one-parameter subgroups of the algebraic torus $T$. (Recall that there is a pairing $X(T) \times Y(T) \rightarrow \mathrm{Z}$ given by taking $\langle\chi, \mu\rangle$ to be the unique integer such that $\chi(\mu(t))=t^{(\chi, \mu\}}$ for all $t \in \mathrm{C}^{\times}$.) First we consider a cone

$$
\sigma=\left\{\lambda_{1} \mu_{1}+\ldots+\lambda_{m} \mu_{m} \mid \lambda_{i} \in \mathrm{R}, \lambda_{i} \geq 0 \text { for every } i\right\}
$$

in $Y(T)_{\mathrm{R}}=Y(T) \otimes \mathrm{Z} \mathrm{R}$ where $\left\{\mu_{1}, \ldots, \mu_{m}\right\}$ are finitcly many oneparameter subgroups (actually we also ask that the cone $\sigma$ have a vertex i.e. $\sigma \cap(-\sigma)=0$.) Next we introduce its dual in $X(T) \mathrm{R}=X(T) \otimes \mathrm{Z} \mathrm{R}$ given by

$$
\sigma^{\vee}=\{\chi \in X(T) R \mid\langle\chi, \mu\rangle \geq 0 \text { for every } \mu \in \sigma\} .
$$

Then we express the monoid $\sigma^{\vee} \cap X(T)$ in terms of a finite number of generators

$$
\sigma^{\vee} \cap X(T)=N \cdot \chi_{1}+\ldots+N \cdot \chi_{N}
$$

(that this can always be done is a consequence of Gordan's lemma [D], $[\mathbf{F}],[\mathrm{O}]$.$) Finally we construct the affine model X_{\sigma}$ by taking, as in the case of the affine plane, the Spectrum of the monoid algebra or equivalently the (scheme theoretic) closure of the image of the map $T \rightarrow$ $\mathrm{C}^{N}$ sending $t$ to $\left(t^{\chi_{1}}, \ldots, t^{\chi_{N}}\right)$.

A toric variety is obtained by glueing together the affine models above along $T$-invariant open subsets. Think for example of the projective plane obtained by glueing three copies of the affine plane along the open orbit and identifying the punctured axes in pairs. Of course, here we are glueing not only the spaces but also the $T$-actions so the actions on our three affine planes have to be compatible. Fortunately the cones introduced earlier allow this compatibility to be expressed in simple terms.

Definition. Let $T$ be an algebraic torus. A collcction $\Sigma$ of cones as above in $Y(T)_{\mathrm{R}}$ is said to be a fan whenever it satisfies the following properties

a) Every face of a cone $\sigma$ in $\Sigma$ also belongs to $\Sigma$.

b) The intersection of any two cones in $\Sigma$ is a face of both of them. 
A fan $\Sigma$ gives rise to a toric varicty $X_{\Sigma}$ obtained by glueing together in turn the pairs of varieties $X_{\sigma_{1}}, X_{\sigma_{2}}$ where $\sigma_{1}, \sigma_{2} \in \Sigma$, along the $T$-invariant open subset $X_{\sigma_{1} \cap \sigma_{2}}$ determined by the common face $\sigma_{1} \cap$ $\sigma_{2}$. It is remarkable that there is a dictionary between combinatorial properties of the fan and geometric properties of $X_{\Sigma}$. For example $X_{\Sigma}$ being compact (resp. smooth) translates into the cones in $\Sigma$ covering all of $Y(T)_{\mathrm{R}}$ (resp. every cone being such that the $\chi_{i}$ 's in (4.1) form a basis of $X(T)$.) Moreover, there is a one-to-one correspondence between the objects of the following three classes:

1) $T$-orbits.

2) $T$-invariant affine open subsets.

3) Cones in the fan.

The correspondence is as follows. A cone $\sigma$ determines the open subset $X_{\sigma}$, and this open set contains a unique orbit $\mathcal{O}_{\sigma}$ which is a closed subset in the relative topology. Note that the codimension of an orbit coincides with the dimension of the corresponding cone. In particular $T$-invariant irreducible divisors $D_{\tau}=\overline{\mathcal{O}_{\tau}}$ correspond to one dimensional cones $\tau$.

It is easy to verify that any smooth toric variety $X_{\Sigma}$ gives a perfect embedding and we associate to it a simplicial complex $\mathcal{C}_{\Sigma}=(V, S)$ defined as follows:

i) $V=\{\tau \in \Sigma: \operatorname{dim} \tau=1\}$

ii) $S=\left\{\Gamma_{\sigma} \mid \sigma \in \Sigma\right\}$ where $\Gamma_{\sigma}=\{\tau \in V \mid \tau \subset \sigma\}$.

It is easy to see that this simplicial complex is equivalent to the one associated to $X_{\Sigma}$ as a perfect embedding.

Recall that the Reisner-Stanley algebra $R_{\Sigma}$ of $\mathcal{C}_{\Sigma}=(V, S)$ is by definition the quotient of the polynomial algebra

$$
\mathrm{Z}\left[x_{v}\right]_{v \in V}
$$

by the relations

$$
\prod_{v \in \Gamma} x_{v}=0
$$

(one for each $\Gamma \notin S$.)

It is useful to think of the $x_{v}$ 's as corresponding to the $T$-invariant divisors $D_{v}$. Then the relation above reflects the fact that $\bigcap_{v \in \Gamma} D_{v}=$ ?. Similarly the monomials $M=\prod x_{v}^{n_{v}}$ determine a unique orbit closure $\overline{\mathcal{O}}=\bigcap_{n_{v}>0} D_{v}$ that we shall call the support of $M$.

Let $\mathcal{O}_{X}\left(D_{v}\right)$ be the line bundle determined by the effective divisor $D_{v}$. Recall that $D_{v}$ will coincide with the zeros of some section $s_{v}$ of $\mathcal{O}_{X}\left(D_{v}\right)$. We choose a $T$-linearization of each $\mathcal{O}_{X}\left(D_{v}\right)$ in such a way that in the induced action on sections $s_{v}$ has weight zero i.e. it is $T$-invariant. 
Proposition. Let $X_{\Sigma}$ be a smooth toric variety. The natural morphism

$$
R_{\Sigma} \rightarrow H_{T}^{*}\left(X_{\Sigma}\right)
$$

sending each $x_{v}$ to the equivariant Chern class of $\mathcal{O}_{X}\left(D_{v}\right)$, is an isomorphism.

Proof: I shall sketch the proof and refer the reader to [BDP] for complete details. The idea is to define compatible filtrations of both rings and to show that the induced homomorphism of graded algebras is an isomorphism.

Choose an ordering of the orbits so that the partial unions $X_{k}=$ $\mathcal{O}_{1} \cup \cdots \cup \mathcal{O}_{k}$ are always open. Next we filter both $R_{\Sigma}$ and $H_{T}^{*}(X)$ as follows:

(1) $F^{k} H_{T}^{*}(X)=\operatorname{ker} H_{T}^{*}(X) \rightarrow H_{T}^{*}\left(X_{k-1}\right)$.

(2) $F^{k} R_{\Sigma}=$ span of the monomials having support in $X-X_{k-1}$.

The graded $k$-th pieces are respectively $H_{T}^{*}\left(X_{k}, X_{k-1}\right) \subset H_{T}^{*}\left(X_{k}\right)$ and the span $R_{k}$ of the monomials with support $\overline{\mathcal{O}_{k}}$. Note that $R_{k}=E_{k}$. $\mathrm{Z}\left[x_{v}\right]_{D_{v} \supset \mathcal{O}_{k}}$ where $E_{k}=\prod_{D_{v} \supset \mathcal{O}_{k}} x_{v}$ maps to the equivariant Euler class of the normal bundle to $\overline{\mathcal{O}_{k}}$. It follows that $R_{k}$ maps isomorphically onto the image of the Thom-Gysin map

$$
H_{T}^{*-2 \operatorname{codim}_{k}}\left(\mathcal{O}_{k}\right) \rightarrow H_{T}^{*}\left(X_{k}, X_{k-1}\right) \subset H_{T}^{*}\left(X_{k}\right)
$$

and we are done.

Another possible approach to this type of result is as follows. Every affine open subset $X_{\sigma}$ retracts $T$-equivariantly to its closed orbit $\mathcal{O}_{\sigma}$. (This can be demonstrated very graphically in the projective case using the compact torus $T_{\text {compact }}=\mathrm{U}(1) \times \cdots \times \mathrm{U}(1)$ and a suitable moment map.) Now a Mayer-Vietoris argument allows us to conclude that

$$
\begin{aligned}
H_{T}^{*}(X) & =\underbrace{\lim _{\sigma \in \Sigma}}_{\sigma \in \Sigma} H_{T}^{*}\left(X_{\sigma}\right)={\underset{\sigma \in}{\stackrel{\lim }{\sigma}} H_{T}^{*}\left(\mathcal{O}_{\sigma}\right)=}= \\
& =\underbrace{\lim }_{\sigma \in \Sigma} H^{*}\left(B T_{\sigma}\right)
\end{aligned}
$$

where we write $T_{\sigma}$ for the stabilizer of any point in $\mathcal{O}_{\sigma}$ and the inverse systems on the right hand side are indexed by the inclusion relations among the cones in the fan. (Note that $T_{\sigma} \subset T_{\eta}$ whenever $\sigma \subset \eta$.) These inverse limits describe explicitly the image of the restriction morphism (3.3). 

by

It is easy to see that the extension of the morphism $X(T) \rightarrow R_{\Sigma}$ given

$$
\chi \mapsto \sum_{\substack{v \in V \\ v=\left(\mu_{v}\right)}}\left\langle\chi, \mu_{v}\right\rangle \cdot x_{v}
$$

can be identified with the natural morphism

$$
H^{*}(B T) \rightarrow H_{T}^{*}\left(X_{\Sigma}\right)
$$

When $X_{\Sigma}$ is projective, the spectral sequence determined by the fibration (2.1) collapses (one argument: the cohomology of base and fibre vanish in odd degrees [BB1-3].) It follows that we can identify the ring $H^{*}\left(X_{\Sigma}\right)$ with the quotient of $H_{\Gamma}^{*}\left(X_{\Sigma}\right)$ by the ideal generated by the strickly positive part of $H^{*}(B T)$. Similar results hold for $X_{\Sigma}$ compact and cohomology with rational coefficients. Thus one reobtains as was to be expected the results of Jurkiewicz and Danilov [D]. But I believe the present approach clarifies the nature of the relations that appear in that description.

Let me describe from this point of view the equivariant approach to the Picard group of $X_{\Sigma}$ that I discovered while working on [B1, Remarks 2.1]. The Picard group of $X$ can be identified with $H^{2}(X)$ [BB1-3], and it is clear from the results above that since $H^{2}(B T)=X(T)$, we have

$$
H^{2}(X)=H_{T}^{2}(X) / X(T)
$$

where

$$
\begin{aligned}
H_{T}^{2}(X) & =\underbrace{\lim }_{\sigma \in \Sigma} H_{T}^{2}\left(\mathcal{O}_{\sigma}\right) \\
& ={\underset{\sigma i m}{\sigma \in \Sigma}}_{\lim _{\sigma}} X\left(T_{\sigma}\right) .
\end{aligned}
$$

But taking characters in the short exact sequence

$$
1 \rightarrow T_{\sigma} \rightarrow T \rightarrow T / T_{\sigma} \rightarrow 1
$$

gives

$$
X\left(T_{\sigma}\right)=X(T) /\left(\sigma^{\perp} \cap X(T)\right)
$$

as was to be expected. Actually, all this can be done purely algebraically using the equivariant Picard group $\operatorname{Pic}_{X}(X)$. This is the group of isomorphism classes of pairs $(L, u)$ consisting of a line bundle $L$ over $X$ and a $T$-linearization of it. The usual theory of line bundles on toric 
varieties can now be realized as follows. Every line bundle $L$ on $X$ has a $T$-linearization and its equivariant Chern class $c(L, u)$ is given by the family $\left(\chi_{\sigma}\right)_{\sigma \in \Sigma} \in H_{T}^{2}(X)$ where each $\chi_{\sigma} \in X\left(T_{\sigma}\right)$ denotes the character describing the isotropy action of the stabilizer $T_{\sigma}$ on the fibre of $L$ over a point in the orbit $\mathcal{O}_{\sigma}$. (This gives an isomorphism of $\operatorname{Pic} r(X)$ and $H_{T}^{2}(X)$.) The cohomology spaces $H^{i}(X, L)$ can now be completely described, as $T$-representations, in terms of the equivariant Chern class $c(L, u)$. This is part of a more general phenomenon that includes the yoga of moment maps. (The knowledgeable reader will recall that the moment map is really a representative for the equivariant Chern class in the de Rham model of equivariant cohomology [AB2].) But this will have to be the object of another paper...

\section{References}

[AB1] M. F. ATTYAH AND R. BOTT, The Yang-Mills equations over Riemann surfaces, Phil. Trans. Royal Soc. London A 308 (1982), 523-615.

[AB2] M. F. ATIYAH AND R. BOTT, The moment map and equivariant cohomology, Topology 23 (1984), 1-28.

[BB1] A. Bialynicki-Birula, Some theorems on actions of algebraic groups, Ann. of Moth. 98 (1973), 480.497.

[BB2] A. BIALYNICKI-BiRULA, On fixed points of torus actions on projective varictics, Bull. Acad. Polon. Sci. Sér. Sci. Math. Astronom. et Phys 22 (1974), 10971101.

[BB3] A. BiaiYvicki-Birlin, Some properties of the decomposition of algebraic varieties determined by actions of a torus, Bull. Acad. Polon. Sci. Sér. Sci. Math. Astronom. et Phys. 24 (1976), 667-674.

[B1] E. Bifet, On complete symmetric varietics, $\Lambda d v$, in Math. 80 (1990), 225-249.

[B2] E. BIFET, Tamagawa numbers and cohomology, in preparation.

[BDP] E. BIfET, C. DE Concini AND C. Procesi, Cohomology of Regular Embeddings, Adv. in Math 82 (1990), 1-34.

[D] V. I. Danilov, The geometry of toric varieties, Russian Math. Surveys 33 (1978), 97-154.

[DP1] C. De CoNcini ANd C. Procesi, Complete symmetric varieties, in "Invariant Theory," Lecture Notes in Math. 996, SpringerVerlag, Berlin and New York, 1983, pp. 1-44. 
[DP2] C. De Concini AND C. Procesi, Complete symmetric varieties II, in "Algebraic Groups and Related Topics," Advanced studies in Pure Math., 1985, pp. 481-513.

[De] P. Deligne, Théorème de Lefschetz et critères de dégénérescence de suites spectrales, Publ. Math. I.H.E.S. 35 (1968), 97-126.

[F] W. Fulton, "Introduction to Toric Varieties," William Roever Lectures in Geometry, Washington Univ, 1989.

[G] V. A. GinzBurg, Equivariant Cohomologics and Kähler's Geometry, Functional Anal. Appl. 214 (1987), 271-283.

[H] W. H. HESSELINK, Uniform instability in reductive groups, J. für Math. 304 (1978), 74-96.

[Hs] W. Hsiang, "Cohomology Theory of Topological Transformation Groups," Springer-Verlag, 1975.

[K] F. C. KIRWAN, "Cohomology of Quotients in Symplectic and Algebraic Geometry," Mathematical Notes, Princeton Univ. Press, 1984.

[N] L. NEss, A Stratification of the Null Cone via the Moment Map, Amer. J. Math. 106 (1984), 1281-1329.

$[\mathrm{O}] \mathrm{T}$. ODA, "Convex Bodies and Algebraic Geometry (An introduction to the theory of toric varieties)," Springer-Verlag, 1987.

[SGA4] M. ARTin, A. GROTHENDIECK AND J. L. VERDiER, "Théorie des Topos et Cohomologie Etale des Schémas (SGA 4)," Lecture Notes in Math. 269, 270 and 305, Springer-Verlag, 1973.

\author{
Department of Mathematics \\ and Institute for Mathematical Sciences \\ State University of New York \\ Stony Brook \\ New York 11794-3651 \\ U.S.A. \\ e-mail: bifet@ math sunysb.edu
}

Rebut el 31 de Març de 1992 